\title{
Applicability of full waveform inversion to sonic logging
}

\author{
Kazuki ISHIKURA $^{1,2}$, Hitoshi MIKADA ${ }^{1}$ and Junichi TAKEKAWA ${ }^{1}$ \\ ${ }^{1}$ Dept. of Civil and Earth Res. Eng., Kyoto University \\ ${ }^{2}$ Now at Mitsui Oil Exploration Co., Ltd. (MOECO)
}

\begin{abstract}
Sonic logging has been widely used for many years to acquire physical properties of hydrocarbon reservoirs. In the early years, the detection of first breaks for acoustic signals travelling along wellbore, and, later, the method was replaced by slowness time coherence (STC) method using full acoustic waveform to accurately estimate the velocity of elastic waves in formation, since the estimation of velocity is important for the identification of fluid contacts such as OWC (Oil-Water Contact), GOC (Gas-Oil Contact), etc. The resolution of existing methods is restricted to 6 inch defined by neighboring receiver distance of logging tool. However, there are significant needs to gain the resolution higher than the current detection of fluid contact locations as well as their variations in time. We, therefore, tried to introduce the method of full-waveform inversion (FWI) as an innovative technique to estimate the elastic velocities with much higher resolution in the subsurface using sonic logging. Although the FWI method has been proven to provide seismic velocities with higher resolution than the other conventional seismic reflection methods, the applicability of FWI to the sonic logging has not been investigated yet, and we first examine if the FWI could be used for the objective to estimate velocities finer than the current limitation of receiver spacing using numerical experiments. Our results show that both GOC and OWC become visible with the resolution higher than the conventional sonic methods whose resolution is 6 inches. We conclude that FWI would be applicable to sonic logging as a high-resolution method.
\end{abstract}

\section{Introducion}

Sonic logging has been widely used for many years to acquire physical properties of hydrocarbon reservoirs. Velocity analysis had been performed by the detection of first break since early years. The method was later replaced by a slowness time coherence (STC) method (Kimball and Marzetta, 1984; Kimball, 1998) using full waveform acquisition, since the estimation of velocity is important for the identification of fluid contacts such as OWC (Oil-Water Contact), GOC (Gas-Oil Contact), etc. The resolution of existing methods is limited to 6 inch defined by the distance of two neighboring receivers of logging tool. However, there are significant needs to gain the resolution higher than the current one.

We, therefore, tried to introduce the method of full-waveform inversion (Tarantola, 1984; 1986) as an innovative technique to acquire high -esolution velocity structure in the subsurface (Vireux, 2009), since the method has been proven as a technique that provides higher resolution than the conventional seismic reflection methods.

Since the applicability of FWI to the sonic logging has not been revealed yet, we examined the applicability of FWI using some numerical models with fluid contacts. Furthermore, we combined FWI with the estimated result using first break as an initial model, and verify the effectiveness of our approach.

\section{Forward modeling in frequency domain}

The discretized equations for the elastic wave equations using a finite-difference approach can be written as

$$
\mathbf{M} \ddot{\tilde{\mathbf{u}}}(t)+\mathbf{K} \tilde{\mathbf{u}}(t)=\tilde{\mathbf{f}}(t)
$$

where $\mathbf{u}(\mathbf{t})$ is the discretized displacement field arranged as a column vector, $\mathbf{M}$ is the mass matrix, $\mathbf{K}$ is the stiffness matrix and $\mathbf{f}(\mathrm{t})$ is source term, also arranged as a column vector.

Eq. 1 is expressed in the time-space domain. We now choose to implement a frequency-domain solution. This allows distinct computational advantages for multisource problems (Pratt 1990). This leads to a straightforward formalism for the inverse methods. Taking the temporal Fourier transform of Eq.1 yields;

$$
\mathbf{K u}(\omega)-\omega^{2} \mathbf{M u}(\omega)=\mathbf{f}(\omega)
$$

where

$$
\mathbf{u}(\omega)=\int_{-\infty}^{\infty} \tilde{\mathbf{u}}(t) e^{-i \omega t} d t
$$

and

$$
\mathbf{f}(\omega)=\int_{-\infty}^{\infty} \tilde{\mathbf{f}}(t) e^{-i \omega t} d t
$$

For simplicity we rewrite Eq.3 as

$$
\mathbf{u}=\mathbf{S}^{-1} \mathbf{f}
$$


where the complex 'impedance' matrix, $\mathbf{S}$, is given by $\mathbf{S}=\mathbf{K}-\omega^{2} \mathbf{M}$.

We performed discretization of the impedance matrix based on cell-based grid set (Min and Yoo, 2004). Eq.5 is solved by using LU decomposition. If the LU decomposition is used to solve Eq.5, the matrix factor can be re-used to solve rapidly the forward problem for any new source vector.

\section{Inverse problem in frequency domain}

We define the misfit vector $\Delta \mathbf{d}=\mathbf{d}_{o b s}-\mathbf{d}_{c a l}(\mathbf{m})$ of dimension $\mathrm{N}$ by the differences at the receiver positions between the recorded seismic data $\mathrm{d}_{\mathrm{obs}}$ and the modeled seismic data $d_{\text {cal }}$ for each source-receiver pair of the seismic survey. And then, we define the misfit function $\mathbf{E}$ as

$$
E=\frac{1}{2} \Delta \mathbf{d}^{*} \Delta \mathbf{d}
$$

where * denotes the transpose conjugate.

The gradient method is a recipe for reducing the L2 norm by iteratively updating the parameter vector according to

$$
\mathbf{m}^{(k+1)}=\mathbf{m}^{(k)}-\alpha^{(k)} \nabla_{p} E^{(k)}
$$

where $\mathrm{k}$ is an iteration number and alpha is a step length chosen to minimize the L2 norm in the direction by the gradient of $\mathrm{E}(\mathrm{m})$. The step length is decided by parabolic fitting (Nush,1979; Vigh and Starr,2008), and gradient of $\mathrm{E}(\mathrm{m})$ is decided by the following equation;

$$
\nabla_{p} E=\operatorname{Re}\left\{\mathbf{F}^{t} \mathbf{v}\right\}
$$

where $\mathbf{F}$ is the columns of which are the virtual source terms for each of the $\mathrm{m}$ physical parameters, $\mathbf{v}$ is the backpropagated wavefield.

\section{Despiking processing (Tukey 53H)}

We need to update model parameters including positions of sources and receivers because sources and receivers of sonic logging tool are in line. However, the calculated gradient has peaks at the positions of sources and receivers (Figure 1a). We iteratively apply despiking method (Tukey $53 \mathrm{H}$ ) (Nikora and Goring, 2002) to this gradient for obtaining smooth change of the gradient (Figure 1b).

\section{Updating model parameters}

Sonic logging tool has the sensitivity on the line where sources and receivers are located, however, it doesn't have the sensitivity on other areas. We compare two kinds of updating strategies. One of them is usual updating and another one is updating using one dimensional gradient along borehole. Figure 2 shows the inversion results from both strategies whereas figure 3 shows the vertical profile of the results along the borehole. The model has a high velocity anomaly at the center of the model. In Figure 2a, we can confirm the false image around the tool. In Figure 2b, we can get the better result than the result in Figure $2 \mathrm{a}$ and the false image doesn't appear. Figure 3 also shows the effectiveness of the strategy of Figure $2 b$. From this experiment, we adopt the updating strategy of Figure $2 b$ in this paper.

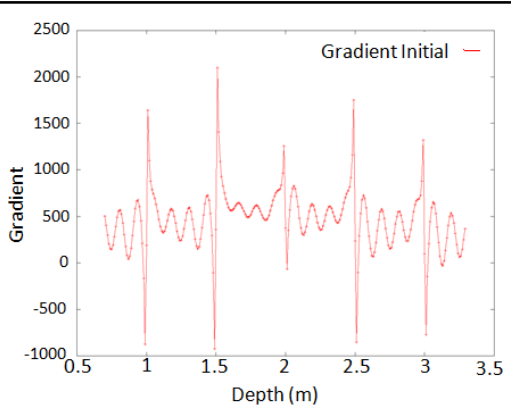

(a)

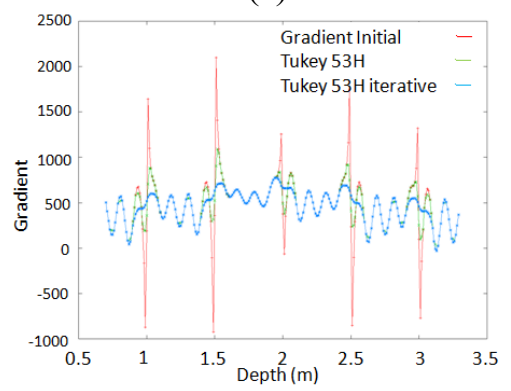

(b)

Figure1 (a) an example of gradient (b) result after adapting of Tukey53H

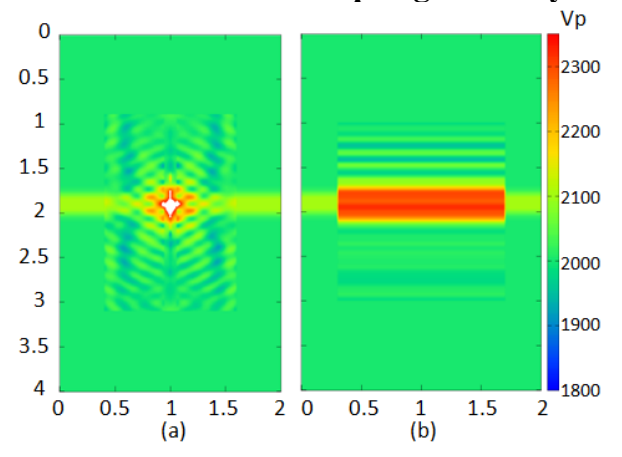

Figure2 (a) usual updating (b) expanded updating

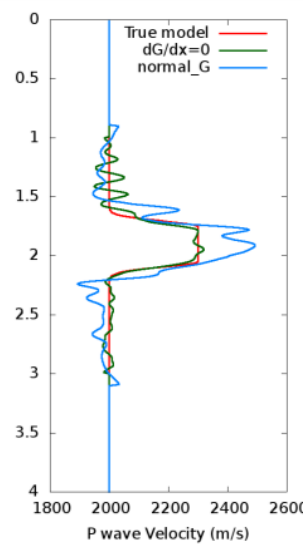

Figure3. vertical plofile of inversion results 


\section{Numerical modeling}

We consider the numerical model shown in Figure 4 . The analysis model has $4 \mathrm{~m} * 1 \mathrm{~m}$, whereas the target area is $3 \mathrm{~m}^{*} 0.8 \mathrm{~m}$ and grid interval is $0.005 \mathrm{~m}$. Sandstone parameters we used are shown in table 1 . The sandstone is saturated with oil and water which are shown in table 2 and we set Oil-Water contact (OWC). Sources and receivers are set in line. The circles and triangles in Figure 4 are sources and receivers, respectively. We use dipole source with frequency components from $20 \mathrm{kHz}$ to $35 \mathrm{kHz}$. Vertical profiles of saturation, Vp and Vs are shown in Figure 5.

\section{Setting initial model}

To determine initial velocity models, we use the conventional velocity analysis using first break. Figure 6 shows initial vertical profiles of Vp and Vs. Green line shows the velocity profile obtained from the travel times of P- and S-waves. On the other hand, red line shows the linearly interpolated profile of green line. We used both velocity models as initial models, and verify the effectiveness of them.

\section{Results}

We compare the inversion results with the step-like model and with the linear interpolation model as shown in the previous section.

First, vertical profiles of velocity structures estimated by using the step-like model as the initial model are shown in Figure 7. And then, the initial residual wave field which is difference of the wave field between the initial model and the true model is shown Figure 8a. The final residual wave field which is difference of the wave field between the model of inversion result and the true model is shown Figure $8 \mathrm{~b}$. The misfit function is shown in Figure 9.

Second, vertical profiles of velocity structures estimated by using the linear interpolation model as the initial model are shown in Figure 10. As is the case with Figure 8, the initial and final residual wave fields are shown in Figure 11a and 11b. The misfit function is shown in Figure 12. Finally, the wavenumber of step-like model, linear interpolation model and true model are shown in Figure 13.

\section{Discussion}

First, in case that we use the step-like model as the initial model, the misfit function decreases on the result of Figure 9. In transition zone, we confirm that the inversion result is different from the true model and $15 \mathrm{~cm}$ sampling distance affects the inversion result. The value of the final residual wave field is smaller than the value of the initial residual wave field. However, the residual from $1 \mathrm{~m}$ to $2 \mathrm{~m}$ can be observed. We think that the inversion result ended up the local minimum because there are clearly reflective surface in the initial model. Second, in case that we use the linear interpolation model as the initial model, the misfit function is smaller than that of step-like model case. And then, the inversion result is very similar to the true model The values of the final residual wave field are almost zero. From Figure 13, the step-like model has high wavenumber components, whereas linear interpolation model does not include high wavenumber components. Therefore, these results mean that we are able to obtain the true velocity structure by using smooth model as an initial model.

\section{Conclusion}

In this study, we examined the applicability of FWI using numerical experiments with the purpose of high-precision of velocity analysis in sonic logging. We summarize what are obtained in this study.

- In sonic logging, we need to update model parameters in the area included sources and receivers. Since the calculated gradient has peaks in the source and receiver positions, we need to use the despiking method for obtaining reliable velocity models.

- The strategy for extrapolating the gradient around the borehole from the calculated gradient on the survey line can improve the accuracy of the inverted velocity model.

- The linearly interpolated initial model provides better results than the step-like initial model.

We conclude that FWI would be applicable to sonic logging as a high resolution technology with appropriate data processing and initial model.

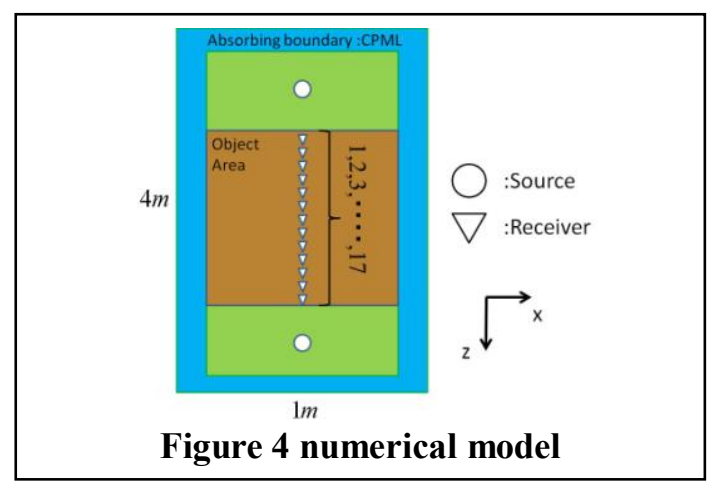




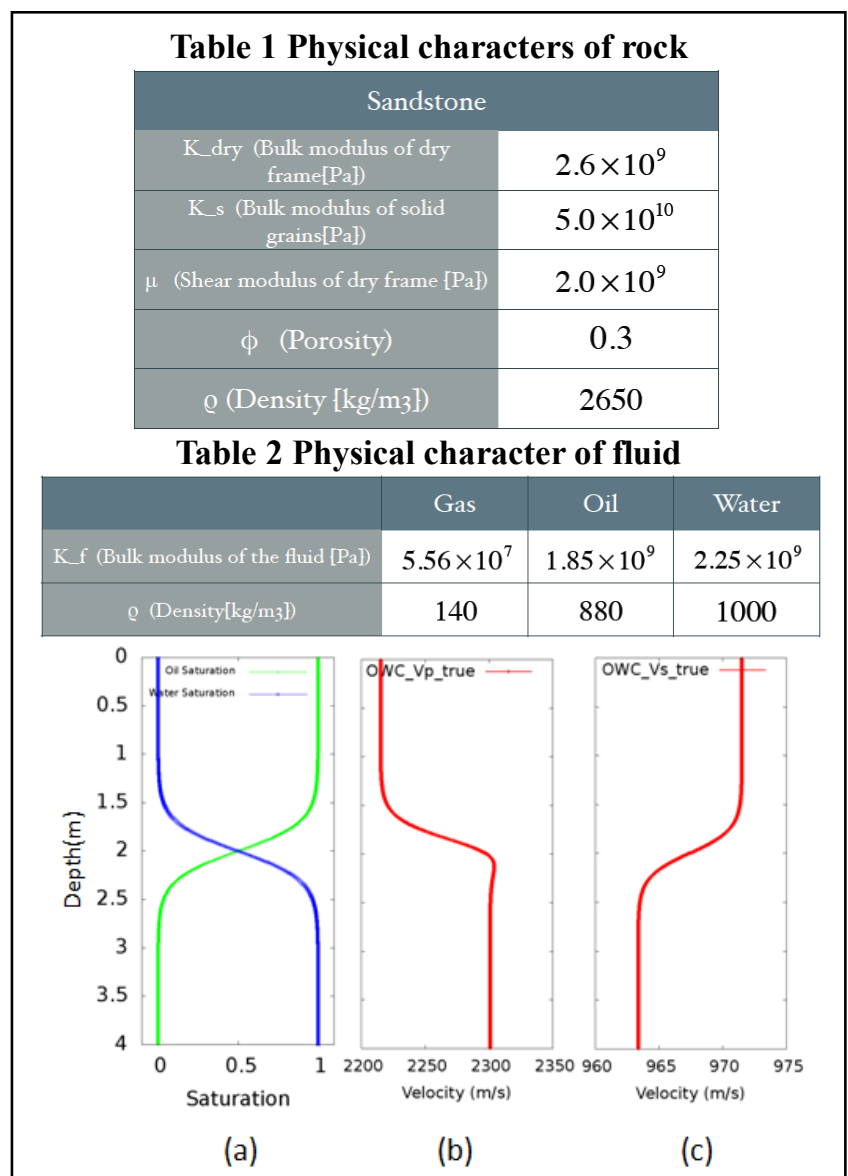

Figure 5 (a) Saturation ratio (b) $P$ wave velocity (c) $S$

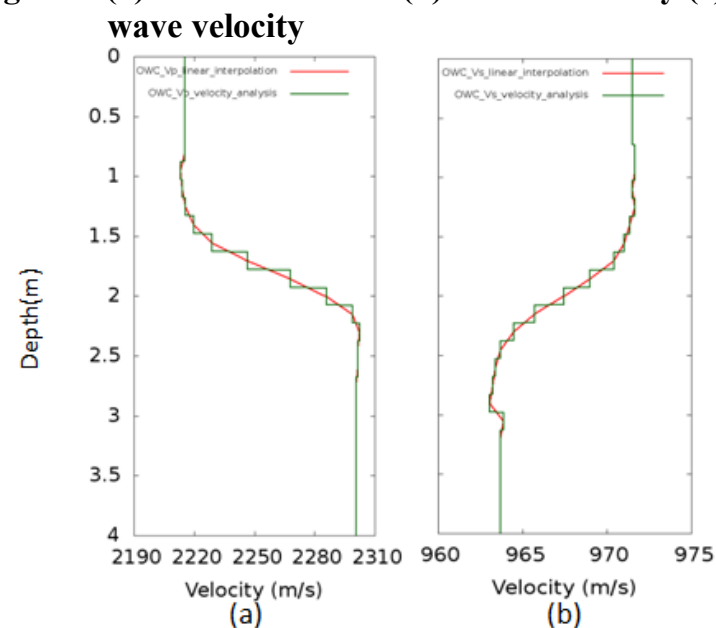

Figure6 Initial model in (a) P-wave and (b) S-wave

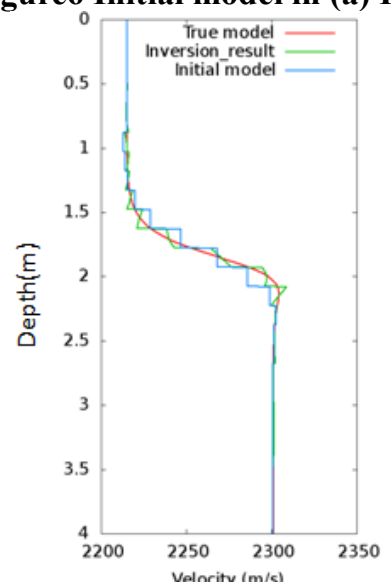

(a)

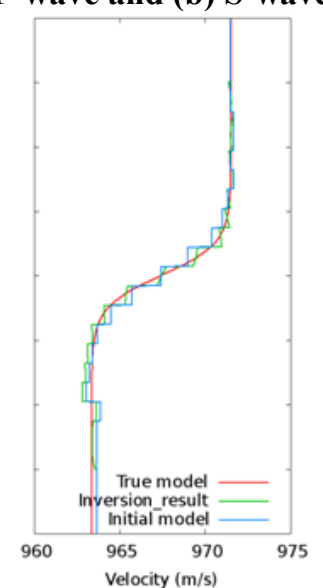

(b)
Figure 7 Inversion results (a) P-wave (b) S-wave

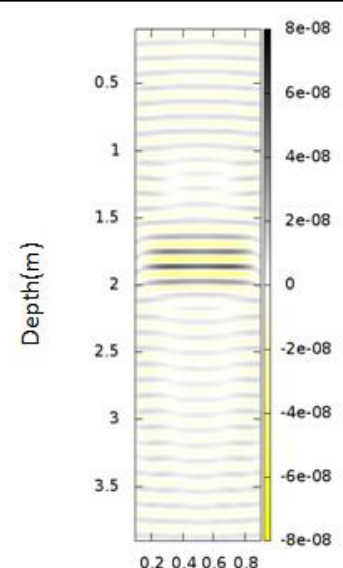

(a)

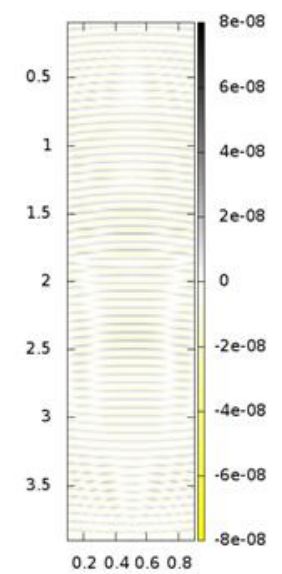

(b)
Figure 8 (a) Initial residual wave field (b) Final residual wave field

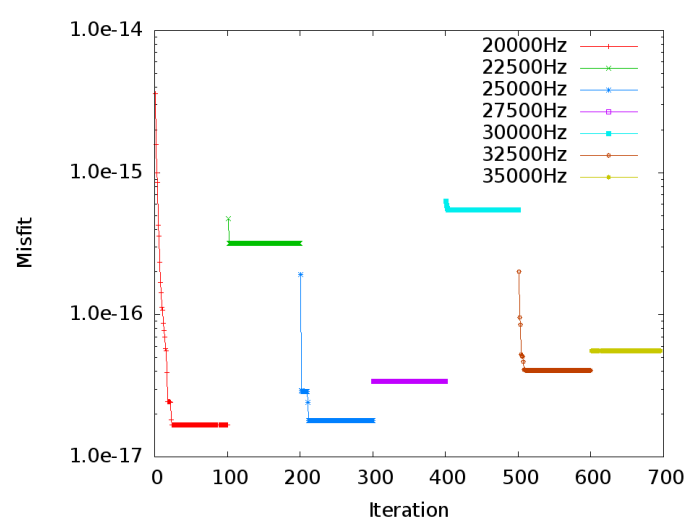

Figure 9 Misfit function

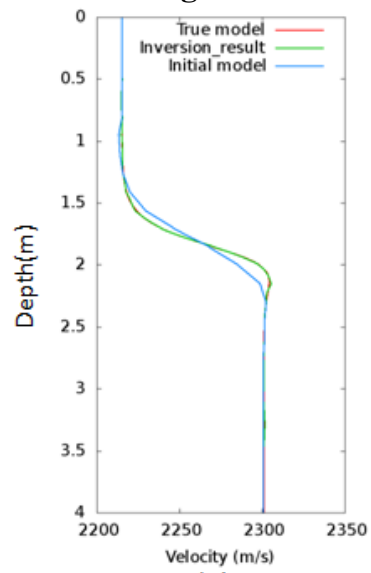

(a)

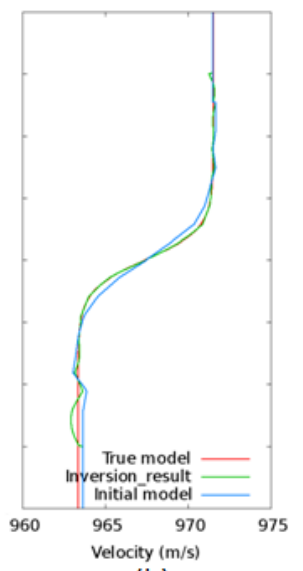

(b)
Figure 10 Inversion results (a) P-wave (b) S-wave

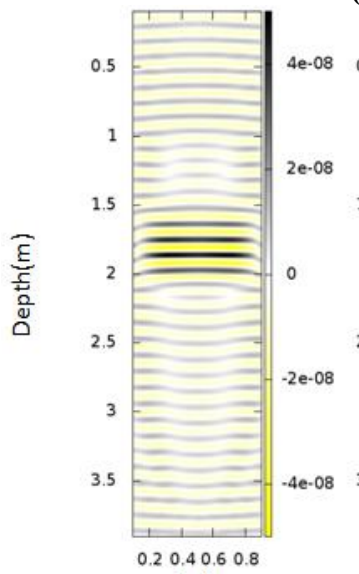

(a)

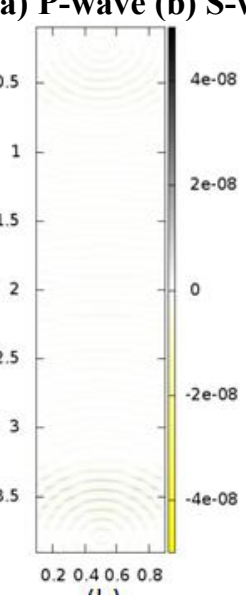

(b)
Figure 11 (a) Initial residual wave field (b) Final residual wave field 


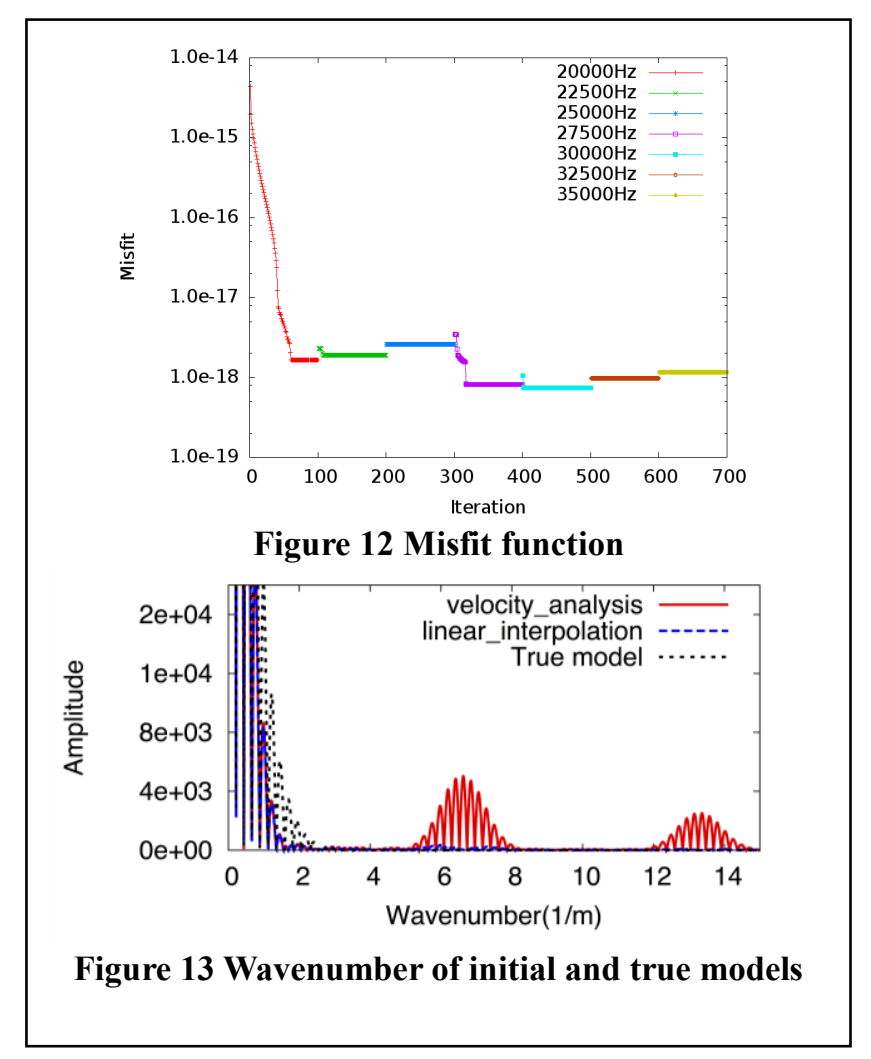

\section{REFERENCES}

1) Goring, D. G., \& Nikora, V. I. (2002). Despiking acoustic Doppler velocimeter data. Journal of Hydraulic Engineering, 128(1), 117-126.

2) Kimball, C. V., \& Marzetta, T. L. (1984). Semblance processing of borehole acoustic array data. Geophysics, 49(3), 274-281.

3) Kimball, C. V. (1998). Shear slowness measurement by dispersive processing of the borehole flexural mode. Geophysics, 63(2), 337-344.

4) Min, D. J., Shin, C., \& Yoo, H. S. (2004). Free-surface boundary condition in finite-difference elastic wave modeling. Bulletin of the Seismological Society of America, 94(1), 237-250.

5) Nash, J. C. (1990). Compact numerical methods for computers: linear algebra and function minimisation. CRC Press.

6) Pratt, R. G. (1990). Frequency-domain elastic wave modeling by finite differences: A tool for crosshole seismic imaging. Geophysics, 55(5), 626-632.

7) Tarantola, A. (1984). Inversion of seismic reflection data in the acoustic approximation. Geophysics, 49(8), 1259-1266.

8) Tarantola, A. (1986). A strategy for nonlinear elastic inversion of seismic reflection data. Geophysics, 51(10), 1893-1903.
9) Vigh, D., \& Starr, E. W. (2008). 3D prestack plane-wave, full-waveform inversion. Geophysics, 73(5), VE135-VE144.

10) Virieux, J., \& Operto, S. (2009). An overview of full-waveform inversion in exploration geophysics. Geophysics, 74(6), WCC1-WCC26. 